\title{
$\left[\mathrm{Cu}_{2} \mathrm{Pt}(\mathrm{npa})_{4} \mathrm{X}_{2}\right]\left(\mathrm{X}=\mathrm{Cl}^{-}, \mathrm{NCS}{ }^{-}\right)$金属串配合物电子传输性质的研究
}

\author{
周沃华 ${ }^{a} \quad$ 陈蓉 ${ }^{a} \quad$ 吴子文 $^{a} \quad$ 丁丹丹 ${ }^{a}$ 徐志广 $a, b$ \\ 许旋*, $a, b, c, d$ 罗一帆 $a, b$ \\ ( ${ }^{a}$ 华南师范大学化学与环境学院 广州 510006) \\ ( ${ }^{b}$ 教育部环境理论化学重点实验室 广州 510006) \\ ( ${ }^{c}$ 广东省高校电化学储能与发电技术重点实验室 广州 510006) \\ ( $d$ 华南师范大学电化学储能材料与技术教育部工程研究中心 广州 510006)
}

\begin{abstract}
摘要 使用密度泛函理论(BP86)结合非平衡态格林函数(NEGF)方法研究金属串配合物 $\left[\mathrm{Cu}_{2} \mathrm{Pt}(\mathrm{npa})_{4} \mathrm{X}_{2}\right]\left(\mathrm{X}=\mathrm{Cl}^{-}(\mathbf{1})\right.$,

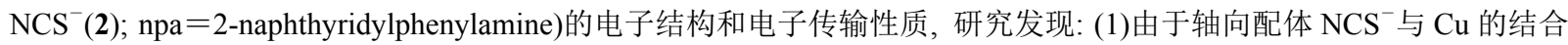
比 $\mathrm{Cl}^{-}$的强, 使配合物 1 的 $\mathrm{Cu}-\mathrm{Cu}$ 键比 $\mathbf{2}$ 的强而 $\mathrm{Cu}-\mathrm{Pt}$ 键比 $\mathbf{2}$ 的弱, 故 $\mathbf{1}$ 的 $\pi^{*}{ }_{\mathrm{Pt} \mathrm{dxz} / \mathrm{yz}}$ 轨道与 $\pi^{*} \mathrm{Cu}-\mathrm{Cu}$ 能级差 $\Delta E$ 比 $\mathbf{2}$ 小. (2) 1 和 2 的传输通道均是 $\beta$ 自旋的 $\pi^{*}$ 轨道, 主要由 $\pi^{*} \mathrm{Cu}-\mathrm{Cu}$ 和 $\pi^{*} \mathrm{Ptdxz} / \mathrm{yz}$ 轨道组合而成. $\Delta E$ 越小 $\pi^{*}$ 越离域, 传输能力越强. 在负偏压下和正偏压小于 $0.15 \mathrm{~V}$ 时, $\mathbf{1}$ 的电流大于 2 ; 但正偏压大于 $0.15 \mathrm{~V}$ 后 2 的 $\beta$ 电流显著高于 1 . (3) 2 具有较好的 整流效应. $\Delta E$ 越大, $\mathrm{Pt} \rightarrow \mathrm{Cu}$ 方向的传输越容易, 整流效应越强. 正偏压下 2 的电流显著大于负偏压下的电流, $0.15 \mathrm{~V}$ 后 2 的整流比比 1 高 10 40 倍. (4)因 $\Delta E_{\beta}<\Delta E_{\alpha}, \alpha$ 自旋通道传输能力小于 $\beta$ 自旋的, 1 和 2 具有良好的自旋过滤效应(高达 $80 \% \sim 99 \%)$.
\end{abstract}

关键词＼cjkstart金属串配合物; 密度泛函理论; 非平衡态格林函数方法; 电子传输; 分子整流器

\section{Electronic Transport Properties of Asymmetric Metal String Complex $\left[\mathrm{Cu}_{2} \mathrm{Pt}(\mathrm{npa})_{4} \mathrm{X}_{2}\right]\left(\mathrm{X}=\mathrm{Cl}^{-}, \mathrm{NCS}^{-}\right)$

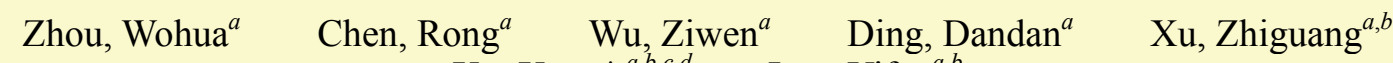 \\ $\mathrm{Xu}, \mathrm{Xuan} *, a, b, c, d \quad$ Luo, Yifan ${ }^{a, b}$ \\ ( ${ }^{a}$ School of Chemistry \& Environment, South China Normal University, Guangzhou 510006) \\ $\left({ }^{b}\right.$ Key Laboratory of Theoretical Chemistry of Environment, Ministry of Education, Guangzhou 510006) \\ ( ${ }^{c}$ Key Laboratory of Electrochemical Technology on Energy Storage and Power Generation in Guangdong Universities, \\ Guangzhou 510006) \\ ( ${ }^{d}$ Engineering Research Center of Materials and Technology for Electrochemical Energy Storage, Ministry of Education of \\ China, South China Normal University, Guangzhou 510006)
}

\begin{abstract}
The asymmetric heterometal string complexes $\left[\mathrm{Cu}_{2} \mathrm{Pt}(\mathrm{npa})_{4} \mathrm{X}_{2}\right]\left(\mathrm{X}=\mathrm{Cl}^{-}(\mathbf{1}), \mathrm{NCS}^{-}(\mathbf{2})\right.$, npa $=2$-naphthyridylphenylamine) are suitable candidates for promising inorganic molecule rectifiers. The electronic structures and transmission properties of them were investigated by theoretical calculation using Density Function Theory BP86 and Non Equilibrium Green's Functions method. The results revealed that: (1) Since the interaction between axial ligand $\mathrm{NCS}^{-}$and $\mathrm{Cu}^{-}$atom is stronger than that of $\mathrm{Cl}-\mathrm{Cu}$, the $\mathrm{Cu}-\mathrm{Cu}$ bond of complex $\mathbf{1}$ is stronger than that in 2 , while its $\mathrm{Cu}-\mathrm{Pt}$ bond is weaker than that in 2. Therefore, complex 1 has smaller energy gap $\Delta E$ between $\pi_{\mathrm{Pt} \mathrm{dxz} / \mathrm{yz}}$ and $\pi_{\mathrm{Cu}-\mathrm{Cu}}^{*}$ orbital compared with that of $\mathbf{2}$. (2) The transmission channels of $\mathbf{1}$ and $\mathbf{2}$ both are $\beta$ spin $\pi^{*}$ orbitals, which are mainly combination of $\pi^{*} \mathrm{Cu}-\mathrm{Cu}$ and $\pi_{\mathrm{Ptdxz} / \mathrm{yz}}^{*}$ orbital. The smaller $\Delta E$, the larger delocalization of $\pi^{*}$ channel, and hence the stronger transmission capacity. When the positive bias is less than $0.15 \mathrm{~V}$ and under negative bias, current of $\mathbf{1}$ is greater than that of $\mathbf{2}$ for the smaller $\Delta E$ of $\mathbf{1}$. However when the positive bias is larger than $0.15 \mathrm{~V}$, current of $\mathbf{2}$ is significantly higher than that of $\mathbf{1}$ for the stronger delocalization of $\pi^{*}$ channel in 2. (3) The complex 2 has better rectifying effect. With a larger $\Delta E$, the transmission of $\mathrm{Pt} \rightarrow \mathrm{Cu}$ direction is easier and the rectifying effect is stronger. Current of $\mathbf{2}$ under the positive bias is significantly greater than that in negative bias. When the bias is larger than $0.15 \mathrm{~V}$, the rectified ratio of 2 is $10 \sim 40$ times larger than 1. (4) With the $\Delta E_{\beta}$ smaller than $\Delta E_{\alpha}$, the transmission capacity of $\alpha$ spin channel is less than $\beta$, and the effect of the spin filter will be stronger for the larger difference between $\Delta E_{\beta}$ and $\Delta E_{\alpha}$. Both 1 and 2 have significant spin filter effect (up to $80 \% \sim 99 \%$ ).
\end{abstract}

*E-mail: xuxuan@scnu.edu.cn; Tel.: 13560091753

Received June 24, 2015; published August 11, 2015.

Supporting information for this article is available free of charge via the Internet at http://sioc-journal.cn.

Project supported by the Natural Science Foundation of Guangdong Province (No. S2012010008763), Ministry of Education and Guangdong Province (No. 2010B090400184), Science and Technology Program of Guangzhou City (No. 2011J4300063).

项目受广东省自然科学资金项目(No. S2012010008763), 广东省教育部产学研项目(No. 2010B090400184)和广州市科技攻关项目(No. 2011J4300063) 资助. 
Keywords metal string complex; density function theory; non equilibrium green's functions; electronic transport; molecule rectifier

\section{1 引言}

分子整流器, 是指在外加偏压下只有单向电流能通 过的分子器件, 由于其理论和实践价值受到广泛关注. 1974 年 Aviram 和 Ratner 最早提出了分子整流器的 $D-\sigma-A$ 分子模型 ${ }^{[1]}$ : 在两个电极之间, 由强的电子给体、 “ $\sigma ”$ 共价桥和强的电子受体三部分组成的分子构成分子 整流器. 以此理论为基础设计的分子整流器大多是共轭 有机物，而金属有机物的整流器少见报道 ${ }^{[2-5]}$.

线性金属串配合物 $\left[\mathrm{M}_{\mathrm{n}} \mathrm{L}_{4} \mathrm{X}_{2}\right]$ 结构新颖, 金属原子呈 线性排列，具有金属-金属键或金属-金属相互作用，四 个桥连配体 $\mathrm{L}$ 螺旋环绕着金属链, 具有独特的电磁性 质, 具有分子电子器件的潜在应用 ${ }^{[6-8]}$. 含不对称金属 链或不对称桥联配体的配合物可能具有分子整流器的 性质, 故彭旭明等 ${ }^{[9]}$ 合成了杂三核金属串配合物 $\left[\mathrm{CuCuM}(\mathrm{npa})_{4} \mathrm{Cl}\right]\left[\mathrm{PF}_{6}\right](\mathrm{M}=\mathrm{Pt}, \mathrm{Pd})$ 并测定其晶体结构和 磁性. 本研究组 ${ }^{[10]}$ 曾用 DFT/BP86 方法研究了电场作用 下该配合物的结构, 发现正负电场下金属原子的自旋密 度的变化不对称, 预测其可能具有自旋整流效应.

电子传输性质是分子电子器件必备的条件. 近五年 来, Mcgrady 和 Peng 等对 $\mathrm{Cr} 、 \mathrm{Co} 、 \mathrm{Ni}$ 等同三核金属串 配合物的电子传输性质进行了模拟计算 ${ }^{[11 ~ 16]}$, 研究集 中在轴向配体为 NCS 的对称金属链的金属串配合物, 对非对称杂核金属串配合物的电子传输研究甚少, 只有 最近对 $\left[\mathrm{Ru}_{2} \mathrm{M}(\mathrm{dpa})_{4}(\mathrm{NCS})_{2}\right](\mathrm{M}=\mathrm{Ni}, \mathrm{Cu})^{[17]}$ 的理论模拟, 发现 $\mathrm{Ni}$ 和 $\mathrm{Cu}$ 原子处在边端导致金属链不对称, 从而产 生分子整流器效应. 目前, 尚未见有关 $\left[\mathrm{CuCuM}(\mathrm{npa})_{4} \mathrm{Cl}\right]$ $\left[\mathrm{PF}_{6}\right](\mathrm{M}=\mathrm{Pt}, \mathrm{Pd})$ 的电子传输性质理论研究的报道, 且 该分子仅有一个轴向配体, 难与两端电极结合. 为便于 金属链与两端电极连接, 本文对具有两端轴向配体 $\mathrm{X}$ 的 非对称配合物 $\left[\mathrm{CuCuPt}(\mathrm{npa})_{4} \mathrm{X}_{2}\right]\left(\mathrm{X}=\mathrm{Cl}^{-}\right.$和 $\left.\mathrm{NCS}^{-}\right)(\mathbf{1}, \mathbf{2})$ 的电子传输性质进行理论研究.

\section{2 模型和计算方法}

为便于轴向配体与两端的金电极相连, 以 $\left[\mathrm{CuCuPt}(\mathrm{npa})_{4} \mathrm{Cl}\right]^{+}$的晶体结构 $\left(\mathbf{1}^{0}\right.$, 图 1 的 a)为基础构建 $\left[\mathrm{CuCuPt}(\mathrm{npa})_{4} \mathrm{Cl}_{2}\right]$ (配合物 $\mathbf{1}^{\prime}$ ).

为节约计算时间, 仿照 Peng ${ }^{[9]}$ 和 McGrady ${ }^{[13]}$ 等的方 法简化桥连配体建立 $\left[\mathrm{CuCuPt}(\mathrm{npa})_{4} \mathrm{X}_{2}\right]\left(\mathrm{X}=\mathrm{Cl}^{-}, \mathrm{NCS}^{-}\right)$ 的计算模型 1 和 2, 采用 ADF2012 $2^{[18-20]}$ 软件的 BP86 方 法进行几何优化, $\mathrm{C} 、 \mathrm{~N} 、 \mathrm{H} 、 \mathrm{~S} 、 \mathrm{Cl}$ 等用双- $\zeta$ 极化基组 (DZP), 而 $\mathrm{Cu} 、 \mathrm{Pt}$ 用三- $\zeta$ 极化基组(TZP), 并使用了 ZORA 标量相对论效应近似. 电子轨道 $1 \mathrm{~s}(\mathrm{C}, \mathrm{N}) 、 2 \mathrm{p}(\mathrm{Cl}$, $\mathrm{S}) 、 3 \mathrm{~d}(\mathrm{Cu})$ 和 $4 \mathrm{f}(\mathrm{Pt})$ 作为核心并使用冻结核进行近似处 理. 为验证分子模型的合理性, 也对未简化的 $1^{0}$ 和 1 '进

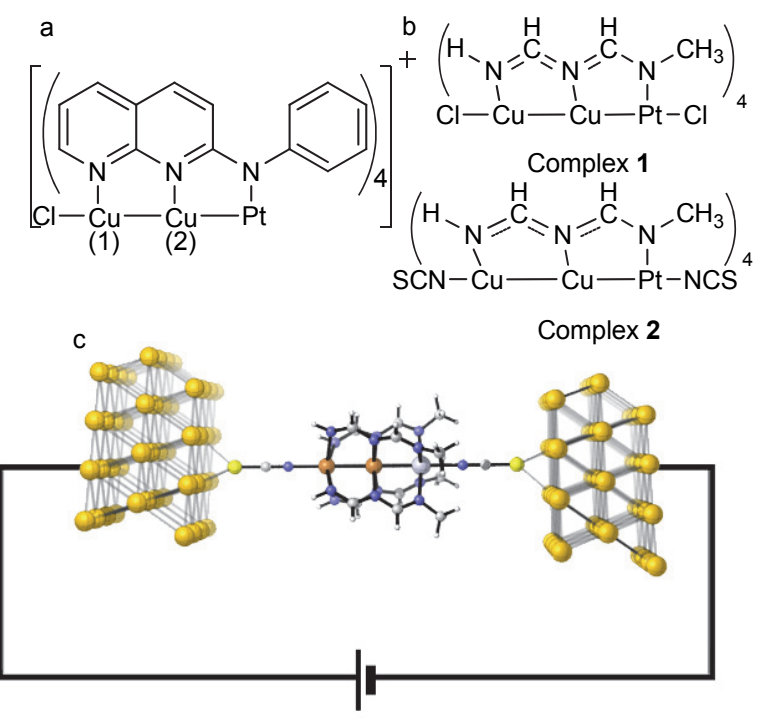

图 $1\left[\mathrm{CuCuPt}(\mathrm{npa})_{4} \mathrm{X}_{2}\right]\left(\mathrm{X}=\mathrm{Cl}^{-}, \mathrm{NCS}^{-}\right)$的结构和计算模型 (a: $[\mathrm{Cu}-$ $\left.\mathrm{CuPt}(\mathrm{npa})_{4} \mathrm{Cl}\right]^{+}$的结构; b: 分子简化计算模型; c: 配合物 2 电场作用模 型)

Figure 1 Computational structure model of $\left[\mathrm{CuCuPt}(\mathrm{npa})_{4} \mathrm{X}_{2}\right]\left(\mathrm{X}=\mathrm{Cl}^{-}\right.$, $\mathrm{NCS}^{-}$). (a: Structure of $\left[\mathrm{CuCuPt}(\mathrm{npa})_{4} \mathrm{Cl}\right]^{+}$; b: The model complexes; c: Model for the complex 2 connecting with the Au electrodes in electric field)

行了几何优化. 所有配合物的优化构型均没有虚频，基 态都是三重态. $1^{0} 、 1^{\prime}$ 的优化构型与实验值很接近，表明 所用的计算方法可行，且增加一个轴向配体 $\mathrm{Cl}^{-}$对配合 物的几何结构影响很小. 除了 $\mathrm{Pt}-\mathrm{Cl}$ 键长差异较大外, 简化模型 1 的几何参数与 $\mathbf{1}$ '的差异均可接受. $\mathrm{Pt}-\mathrm{Cl}$ 键 长远大于 $\mathrm{Cu}-\mathrm{Cl}$ 键, 与实验合成的 $\left[\mathrm{CuCuPt}(\mathrm{npa})_{4} \mathrm{Cl}\right]^{+}$ 中因苯环的空间位阻使 $\mathrm{Cl}^{-}$不能与 $\mathrm{Pt}$ 配位 ${ }^{[9]}$ 的现象相符. 因此，简化模型是可行的.

依据 1 和 $\mathbf{2}$ 的基态优化构型, 采用非平衡态格林函 数(NEGF) ${ }^{[21]}$ 结合 LSDA-CA 方法在 OpenMX 3.7 程序 ${ }^{[22]}$ 上计算配合物 $\mathbf{1}$ 和 $\mathbf{2}$ 的电子传输性质, 基组采用完全相 对论赝势(VPS)和赝原子轨道 $(\mathrm{PAO})^{[23]}$. PAO 函数是半径 函数和球面调和函数的积, 各原子的截断半径 $r_{\mathrm{c}}$ 和 $\mathrm{s}, \mathrm{p}$, $\mathrm{d}$ 轨道的原始轨道的数目见支持信息 S1. 设置电子温度 $300 \mathrm{~K}$, 截止能量 $150 \mathrm{Ry}$. 施加 $\mathrm{Cu} \rightarrow \mathrm{Pt}$ 方向的偏压(正偏 压)和 $\mathrm{Pt} \rightarrow \mathrm{Cu}$ 方向的偏压(负偏压). 散射区域为三明治 结构, 包括金属串配合物和 $4 \times 4 \times 3$ 层 $\mathrm{Au}(111)$ 面组成 的漏电极和干电极. 针原子 $\mathrm{Cl}$ 或 $\mathrm{S}$ 原子正对 $\mathrm{Au}(111)$ 面 的一个空穴位置, 与三个 $\mathrm{Au}$ 原子的距离为 $2.52 \AA$.

\section{3 结果与讨论}

\section{1 电子结构}

因轴向配体 $\mathrm{NCS}^{-}$的 $\sigma$ 给电子能力比 $\mathrm{Cl}^{-}$强 ${ }^{[24]}, 2$ 中 
轴向的 $\mathrm{N}-\mathrm{Cu}$ 键比 1 的 $\mathrm{Cl}-\mathrm{Cu}$ 键强, $\mathrm{N}-\mathrm{Cu}$ 键长 2.031 $\AA$ 远比 $\mathrm{Cl}-\mathrm{Cu}$ 键的 $2.347 \AA$ 短, $\mathrm{N}-\mathrm{Cu}$ 键 Mayer 键级 0.5408 比 $\mathrm{Cl}-\mathrm{Cu}$ 键的 0.5107 大. 因此, 由表 1 可见, $\mathbf{1}$ 的 $\mathrm{Cu}-\mathrm{Cu}$ 键长小于 2 , 而 $\mathrm{Cu}-\mathrm{Pt}$ 键长大于 2 . 由 $\mathbf{1}$ 的 $\mathrm{Cu}-\mathrm{Cu} 、 \mathrm{Cu}-\mathrm{Pt}$ 键级分别为 0.3788 和 0.3687 和 2 的 $\mathrm{Cu}-\mathrm{Cu} 、 \mathrm{Cu}-\mathrm{Pt}$ 键级分别为 0.3121 和 0.3965 可见, $\mathbf{1}$ 的 $\mathrm{Cu}-\mathrm{Cu}$ 相互作用比 2 的强, $\mathrm{Cu}-\mathrm{Pt}$ 相互作用比 $\mathbf{2}$ 的 弱, 且零电场下 1 中金属链更离域. 如图 2 前线分子轨 道能级图所示: 1 和 2 都具有两个单电子, 单占轨道均为 $\alpha$ 自旋的 $\sigma^{*}$ 轨道和 $\delta^{*} \mathrm{Cu}$-Cu 轨道. 化学键越强, 其成键分 子轨道能越低, 反键分子轨道能越高. 因此, $\mathbf{1}$ 的 $\pi^{*}{ }_{\mathrm{Cu}-\mathrm{Cu}}$ 轨道能比 2 高, 而 $\pi_{\mathrm{Pt} \mathrm{dxz} / \mathrm{yz}}^{*}$ (以 $\mathrm{Pt}$ 为主的 $\mathrm{Pt}-\mathrm{Cu}$ 反键 $\pi$ 轨道)轨道能比 2 低.

表 1 配合物的键长 $(\AA)^{a}$

Table 1 Computed bond lengths $(\AA)$ of complexes

\begin{tabular}{ccccc}
\hline Complex & $\mathrm{X}-\mathrm{Cu}(1)$ & $\mathrm{Cu}(1)-\mathrm{Cu}(2)$ & $\mathrm{Cu}(2)-\mathrm{Pt}$ & $\mathrm{Pt}-\mathrm{X}$ \\
\hline $\mathbf{1}$ & 2.347 & 2.553 & 2.531 & 2.672 \\
$\mathbf{2}$ & 2.031 & 2.561 & 2.512 & 2.240 \\
$\mathbf{1}^{\prime}$ & 2.387 & 2.496 & 2.494 & 2.563 \\
& 2.392 & 2.491 & 2.488 & - \\
$\mathbf{1}^{\mathbf{0}}$ & $(2.404)$ & $(2.489)$ & $(2.436)$ & - \\
\hline
\end{tabular}

${ }^{a}$ Experimental values in parentheses.

借鉴 Kondo 等的方法 ${ }^{[25,26]}$ 分析分子轨道对应传输 谱图峰. 由图 3 的零偏压下传输谱图可见, $\mathbf{1}$ 有一 $\beta$ 自旋 传输谱峰略低于 $\mathrm{Au}$ 电极 Fermi 能级, 2 有一 $\beta$ 自旋谱峰 稍高于 $\mathrm{Au}$ 电极 Fermi 能级. 由图 2 可见: 零偏压下配合 物 1 在 Fermi 能级附近有 $\pi^{*} \mathrm{Pt} \mathrm{d} z z \mathrm{yz} z$ 轨道和 $\delta^{*}$ 轨道; 配合 物 2 在 Fermi 能级附近有 $\pi^{*}{ }^{*} \mathrm{Ptd} z z \mathrm{yz} z$ 轨道和 $\delta_{\mathrm{Cu}-\mathrm{N}}^{*}$ 轨道. 因 $\delta^{*}$ 和 $\delta^{*} \mathrm{Cu-N}$ 轨道分别由金属的 $\mathrm{d} x y 、 \mathrm{~d} x^{2}-y^{2}$ 轨道组成, 轨 道的伸展方向与 $Z$ 轴垂直, 故不可能是沿 $Z$ (金属轴)方 向传输的主要通道; 传输通道可能是以 $\pi^{*} \mathrm{Pt} d z z / y z$ 轨道为 主的轨道. 因此指认 1 和 2 的传输通道为 $\pi^{*}$, 该通道以 $\pi_{\mathrm{Pt} \mathrm{d} z z \mathrm{yz}}^{*}$ 为主, 还含少量的 $\pi^{*}{ }_{\mathrm{Cu}-\mathrm{Cu}}$ 和金电极的成份.

由图 4 分析分子轨道 $\pi^{*} \mathrm{Pt} \mathrm{dxzyz}$ 和 $\pi_{\mathrm{Cu}-\mathrm{Cu}}^{*}$ 与 $\mathrm{Au}$ 电极 相连后形成传输通道 $\pi^{*}$ 的成因, 可以定性地看作是由 $\mathrm{Cu}-\mathrm{Cu}$ 部分与 $\mathrm{Pt}$ 部分的轨道 $\left(\pi^{*}{ }_{\mathrm{Cu}-\mathrm{Cu}} \text { 和 } \pi_{\mathrm{Pt} \mathrm{d} z z / y z}\right)^{*}$ 的线性 组合. 由于电子-电子排斥的存在, 轨道存在自旋分 裂 ${ }^{[15]}$. 排斥作用越大, 则轨道自旋分裂能也越大, 自旋 极化也越大. $\mathrm{Cu}$ 的 $3 \mathrm{~d}$ 轨道能量比 $\mathrm{Pt}$ 的 $5 \mathrm{~d}$ 轨道能量低, $3 \mathrm{~d}$ 轨道较为收缩, $3 \mathrm{~d}$ 电子-电子排斥作用大, 故配合物 $1 、 2$ 的 $\pi_{\mathrm{Cu}-\mathrm{Cu} \alpha / \beta}^{*}$ 能级分裂比 $\pi_{\mathrm{Pt} \mathrm{dxz} / y z}^{*}$ 轨道显著. 且 2 的 $\pi^{*}{ }_{\mathrm{Cu}-\mathrm{Cu}} \alpha / \beta$ 能级分裂(约 $0.33 \mathrm{eV}$ )比 $\mathbf{1}$ 的(约 $0.29 \mathrm{eV}$ )大, 而 $\pi^{*}{ }_{\mathrm{Ptd} d z / y z} \alpha / \beta$ 能级分裂(约 $0.03 \mathrm{eV}$ )比 $\mathbf{1}$ 的(约 $0.10 \mathrm{eV}$ )小.

传输通道 $\pi^{*}$ 的离域性将决定电子传输的难易, 离域 程度越高传输越容易. $\alpha 、 \beta$ 自旋 $\pi^{*}$ 的离域性由 $\pi^{*}{ }^{*} \mathrm{Ptd} z z / y z$ 与 $\pi^{*} \mathrm{Cu}$-Cu 轨道的能级差 $\Delta E_{\alpha}$ 和 $\Delta E_{\beta}$ 决定, 能级差越小, 轨道组合越有效, 通道离域程度越高, 电子的传输越容
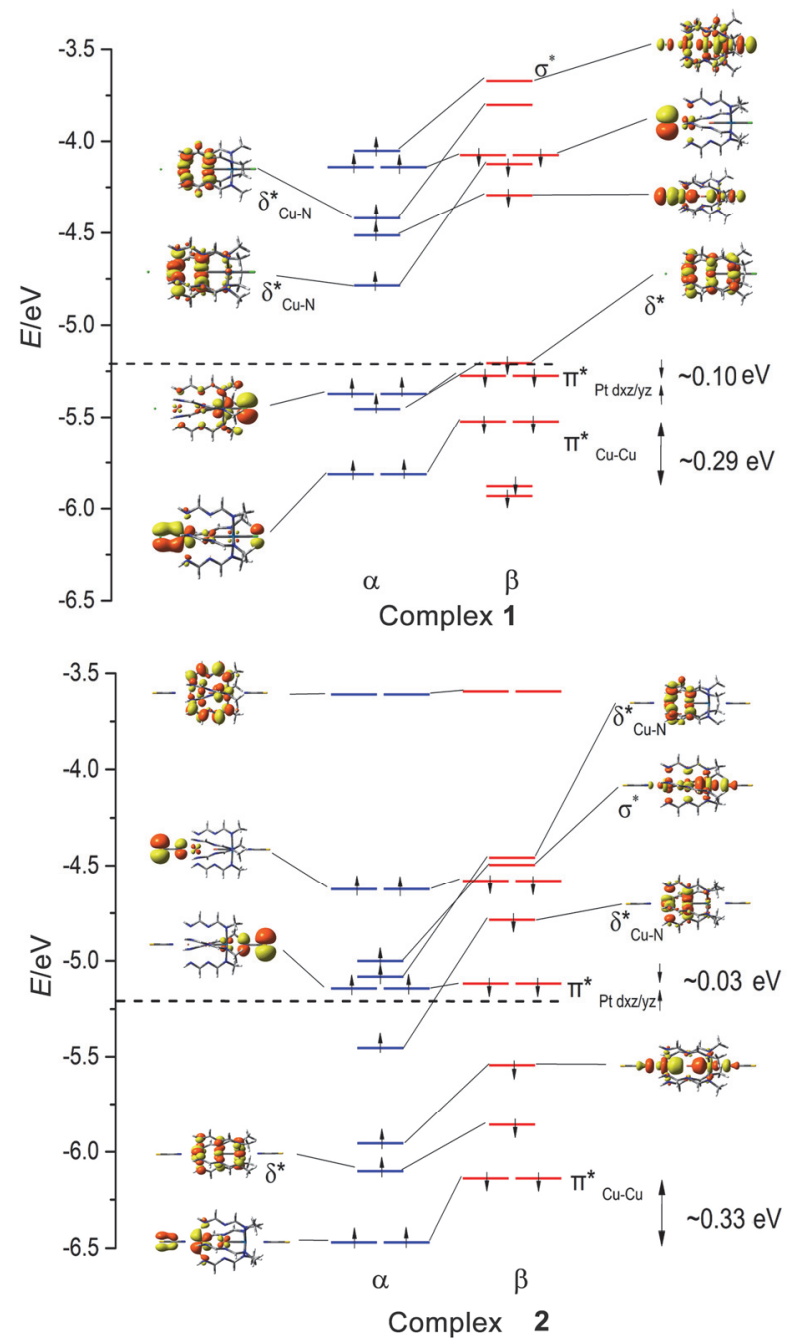

图 2 配合物 1 和 $\mathbf{2}$ 的前线分子轨道(虚线表示金电极的费米能级) Figure 2 Molecular orbital level of an isolated complex $\mathbf{1}$ and $\mathbf{2}$ and the some MOs. The dotted line indicates the Fermi energy of the gold electrode

易. 但 $\Delta E$ 还影响体系的整流效应, $\Delta E$ 越大, $\mathrm{Pt} \rightarrow \mathrm{Cu}$ 方向 电子越容易通过而 $\mathrm{Cu} \rightarrow \mathrm{Pt}$ 方向的电子越难通过，即整 流效应越大. 因 1 的 $\mathrm{Cu}-\mathrm{Cu}$ 键比 2 的强, $\mathrm{Cu}-\mathrm{Pt}$ 键比 2 的弱, 使 $\mathbf{1}$ 的 $\pi^{*} \mathrm{Cu}-\mathrm{Cu}$ 轨道能比 $\mathbf{2}$ 的高, $\pi_{\mathrm{Pt} \mathrm{dxz} z y z}^{*}$ 轨道能比 2 的低, 故 1 的 $\Delta E$ 比 $\mathbf{2}$ 的小. 因此, 2 的整流效果可能比 1 的强, 而 1 的电子传输可能比 $\mathbf{2}$ 的强. 此外 1 和 $\mathbf{2}$ 中 $\Delta E_{\beta}<\Delta E_{\alpha}$ ，故自旋通道 $\pi_{\beta}^{*}$ 的离域性大于 $\pi_{\alpha}^{*}, \beta$ 自旋的电 子比 $\alpha$ 自旋的更容易传输, 故 1 和 2 都具有良好的自旋 过滤效应.

\section{$3.2 I-V$ 曲线}

Peng 等 ${ }^{[9]}$ 认为因 $\mathrm{Pt}$ 的 $5 \mathrm{~d}$ 轨道比 $\mathrm{Cu}$ 的 $3 \mathrm{~d}$ 轨道能量 高, $\left[\mathrm{CuCuPt}(\mathrm{npa})_{4} \mathrm{Cl}\right]\left[\mathrm{PF}_{6}\right]$ 可能具有分子整流器的性质. 本课题组 ${ }^{[10]}$ 曾应用 BP86 方法研究发现：随 $\mathrm{Cu} \rightarrow \mathrm{Pt}$ 方向 电场增大 $\mathrm{Cu}$ 的自旋密度减小而 $\mathrm{Pt}$ 的自旋密度单调增大 明显; 而随着 $\mathrm{Pt} \rightarrow \mathrm{Cu}$ 方向电场增大原子的自旋密度仅 略为增大，当电场增大至 $1.543 \mathrm{~V} \cdot \mathrm{nm}^{-1}$ 时 $\mathrm{Cu} 、 \mathrm{Pt}$ 的自 


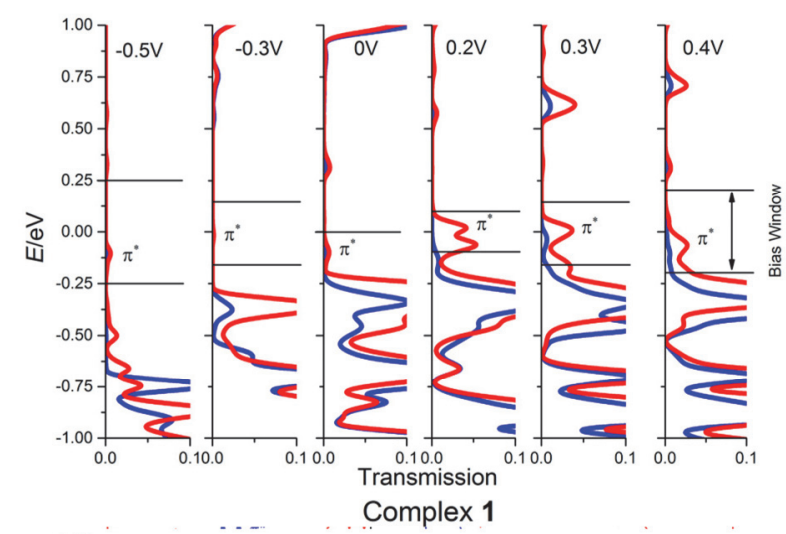

$0.15 \mathrm{~V}$ 之后 1 出现 $\beta$ 自旋平台电流, 大小约 $60 \mathrm{nA}$; 在负 偏压作用下, 电流先增大后减小, 出现了负微分电阻效 应 $^{[27]}$, 在 $-0.3 \mathrm{~V}$ 时取得极大值. 负偏压下, 2 的电流小 于 1 , 这与 $\mathbf{2}$ 的 $\Delta E$ 大于 1 使其电子传输能力弱于 1 的结 论一致. 此外, 2 在负偏压时也出现负微分电阻效应, 在 $-0.1 \mathrm{~V}$ 处取得极大值, $-0.3 \mathrm{~V}$ 处取得极小值. 而在正 偏压作用下, 2 的 $\beta$ 自旋电流呈递增趋势, 在小于 $0.15 \mathrm{~V}$ 时电流小于 1 , 但从 $0.15 \mathrm{~V}$ 之后电流显著大于 1 , 这可能 是含 $\mathrm{NCS}^{-}$共轭配体的 2 的传输通道 $\pi^{*}$ 的轨道分布受电 场作用变得更离域的缘故(见支撑材料 S2).

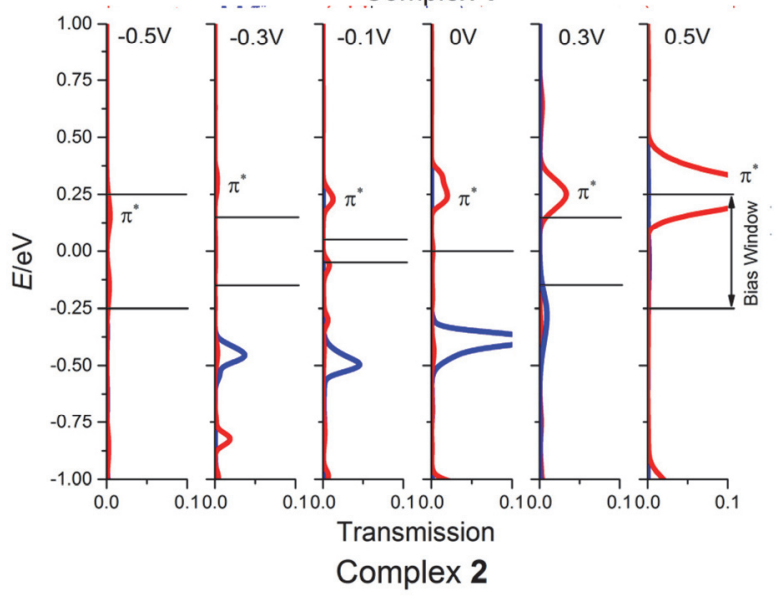

图 3 配合物 1 和 2 的传输谱图(Fermi 能级设为 $0 \mathrm{eV}$, 蓝色为 $\alpha$ 自旋, 红色为 $\beta$ 自旋)

Figure 3 Transmission of complex $\mathbf{1}$ and $\mathbf{2}$ (Fermi energy is set to be 0 $\mathrm{eV}$. Blue and red line denote the spin- $\alpha$ and spin- $\beta$ manifolds, respectively.)
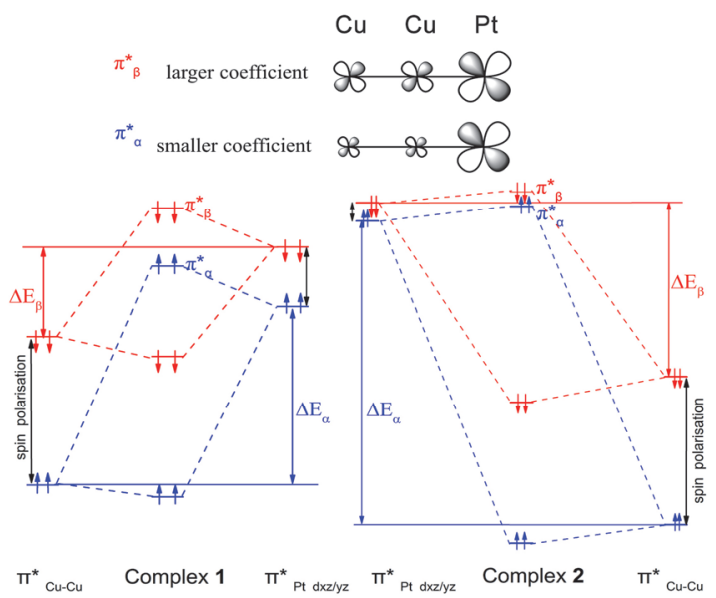

图 $4 \pi$ 通道的成因示意图

Figure 4 The formation and delocalization of $\pi^{*}$ channels

旋密度不再变化, 出现平台, 预测其具有自旋整流器的 潜在应用. 为更深入了解 $\mathrm{CuCuPt}(\mathrm{npa})_{4} \mathrm{Cl}_{2}$ 和 $\mathrm{CuCuPt}(\mathrm{npa})_{4}(\mathrm{NCS})_{2}$ 自旋整流器的性质, 对其 $I-V$ 曲线 进行分析.

从 $I-V$ 曲线(图 5)可见, 由于 $\Delta E_{\beta}<\Delta E_{\alpha}, 1$ 和 2 中 $\beta$ 自旋的电流确实都显著大于 $\alpha$ 自旋. 在正偏压作用下,

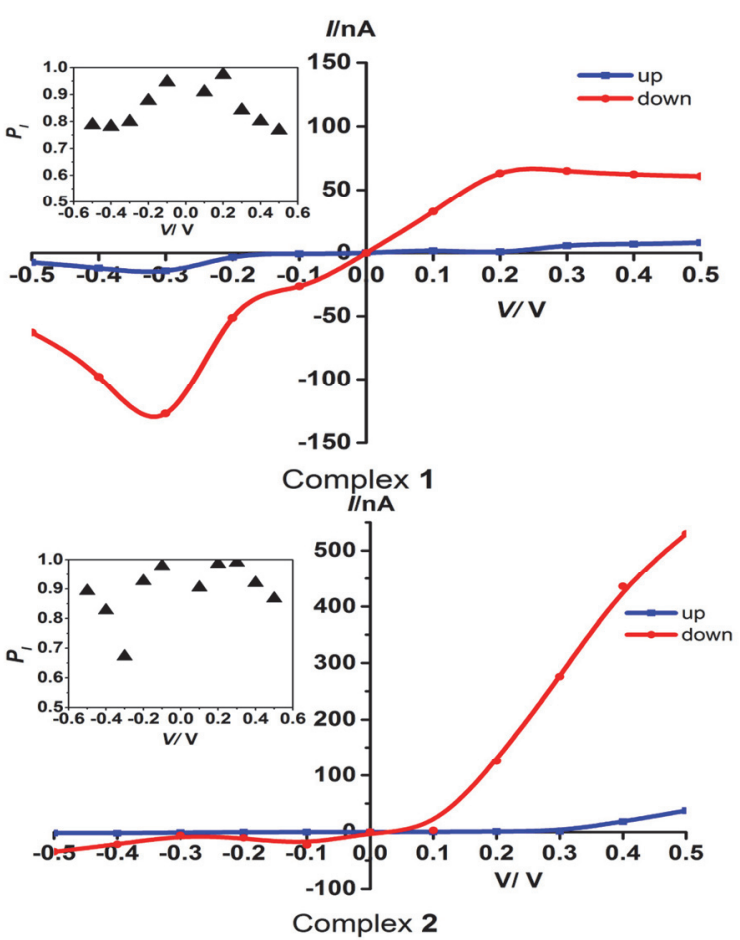

图 $5 I-V$ 曲线图和自旋极化率 $P_{I}$ 随偏压变化图

Figure 5 Computed current-voltage characteristics and spin polarization vs bias voltage

将整流比 $R$ 定义为相同数值正、负偏压下总电流绝 对值 $\left(I=\mid I_{\mathrm{up}}+I_{\mathrm{down}}\right)$ 的比值 $R(V)=I(V) / I(-V)$, 得到 $R-V$ 图 (图 6). 整流比 $R$ 是体系的单向导电性大小的直接表现, $R$ 越大则单向导电性越显著. 在偏压 $0.15 \mathrm{~V}$ 后 2 的整流 比 $R$ 是 1 的 10 40 倍, 证实了 2 的整流效应远大于 1 . 这 与 $I-V$ 曲线中当正偏压大于 $0.15 \mathrm{~V}$ 时 2 的电流比 1 更大 的现象相吻合, 也与本课题组 ${ }^{[10]}$ 对 $\left[\mathrm{CuCuPt}(\mathrm{npa})_{4} \mathrm{Cl}\right]^{+}$体 系的自旋密度随电场变化得到的结论一致.

自旋过滤效应 $\left(P_{I}=\left(I_{\text {down }}-I_{\text {up }}\right) /\left(I_{\text {down }}+I_{\text {up }}\right) \times 100 \%\right)$ 可以反映体系中自旋电流对总电流的贡献 ${ }^{[28]}$. $P_{I}$ 随偏压 的变化可见图 4 插图, $P_{I}$ 越大, $\beta$ 与 $\alpha$ 自旋的电流之差越 大. 配合物 1 和 2 的 $P_{I}$ 值主要集中在 $80 \% \sim 99 \%$ 范围， 都具有良好的自旋过滤效应. 这说明电流主要由自旋向 下的 $\beta$ 电流贡献, 而自旋向上的 $\alpha$ 电流则很弱, 这与前 文分析 $\Delta E_{\beta}<\Delta E_{\alpha}$ 所得结论一致. 偏压较低时 $P_{I}$ 较大, 
配合物 1 的 $P_{I}$ 在 $0.15 \mathrm{~V}$ 时达到 $97.34 \%$, 配合物 2 的 $P_{I}$ 在 $0.3 \mathrm{~V}$ 时达到 $99.03 \%$. 随着正偏压增大, $P_{I}$ 有所降低.

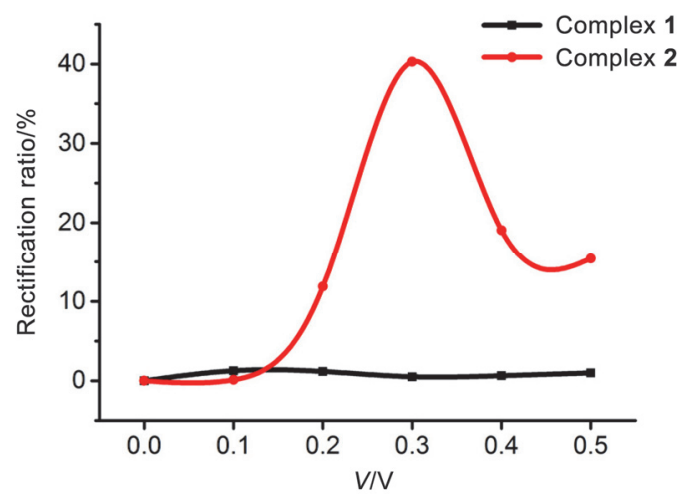

图 6 配合物 1 和 $\mathbf{2}$ 的整流比与偏压 $(V)$ 的关系

Figure 6 Rectification ration vs. voltage $(V)$ of complex 1 and $\mathbf{2}$

\section{3 传输谱图}

当分子器件施加偏压后, 使两电极的 Fermi 能级相 对分子的 Fermi 能级上下移动而形成偏压窗口(见图 3 中两条横线之间的能量区域“bias window”), 分子器件 的导电性质则由偏压窗口内的电子传输谱所决定. 由图 3 的传输谱可见, 在计算的偏压范围内, 1 和 2 的 $\pi^{*}$ 通道 处于偏压窗口内或附近. 正偏压下 1 的 $\pi^{*}$ 从 $0.15 \mathrm{~V}$ 开 始峰强变化不明显, 这与图 5 正偏压下出现平台电流现 象一致; 2 的 $\pi^{*}{ }_{\beta}$ 峰强随着正偏压增大而增强, 并且从 0.3 $\mathrm{V}$ 开始进入偏压窗口, 这与 2 的 $\beta$ 电流当正偏压大于 $0.15 \mathrm{~V}$ 时电流比 1 的大且随正偏压增大而增大的结论一 致. 负偏压下, 配合物 $\mathbf{1}$ 的 $\pi^{*}{ }_{\beta}$ 峰虽始终处于偏压窗口 内, 但随负偏压增大而减小; 而配合物 2 的 $\pi^{*}{ }_{\beta}$ 峰从 $-0.5 \mathrm{~V}$ 才进入偏压窗口, 这与负偏压下 2 的电流比 1 小一致, 也与 2 的负微分现象一致. 1 和 2 的偏压窗口内 的 $\alpha$ 自旋电子传输谱明显比 $\beta$ 自旋的小, 几乎可以忽略 不计，也表明其具有自旋过滤效应.

\section{4 结论}

对 $\left[\mathrm{CuCuPt}(\mathrm{npa})_{4} \mathrm{X}_{2}\right]\left(\mathrm{X}=\mathrm{Cl}^{-}, \mathrm{NCS}^{-}\right)$配合物 $\mathbf{1 , 2}$ 的 密度泛函研究得到: 1 和 2 的电子传输通道都是 $\beta$ 自旋的 $\pi^{*}$ 轨道, 主要由 $\pi^{*}{ }_{\mathrm{Cu}-\mathrm{Cu}}$ 和 $\pi_{\mathrm{Ptd} x z / y z}$ 轨道组合而成. 含离域 轴向配体的 2 具有较好的整流效应和自旋过滤效应 $(80 \% \sim 99 \%) . \pi^{*} \mathrm{Cu}-\mathrm{Cu}$ 与 $\pi^{*} \mathrm{Ptd} x z / y z$ 能级差 $\Delta E$ 越小, $\pi^{*}$ 轨道越 离域, 传输能力越强; $\Delta E$ 越大, 整流效应越强; $\Delta E_{\beta}$ 越小
于 $\Delta E_{\alpha}$ ，自旋过滤效应越强. 因 1 的 $\Delta E$ 比 2 的小, 2 的 整流效应比 1 的强, $0.15 \mathrm{~V}$ 后 2 的整流比 $10 \sim 40$ 倍高于 1; 在负偏压下和正偏压小于 $0.15 \mathrm{~V}$ 时, $\mathbf{1}$ 的电流大于 2 , 但正偏压大于 $0.15 \mathrm{~V}$ 时 2 的 $\beta$ 电流显著高于 $\mathbf{1}$.

\section{References}

[1] Aviram, A.; Ratner, M. A. Chem. Phys. Lett. 1974, 29, 277.

[2] Lin, S. Y.; Chen, I. W. P.; Chen, C. H.; Hsieh, M. H.; Yeh, C. Y.; Lin, T. W.; Chen, Y. H.; Peng, S. M. J. Phys. Chem. B 2004, 108, 959.

[3] Braunstein, P.; Frison, C.; Oberbeckmann-Winter, N.; Morise, X.; Messaoudi, A.; Benard, M.; Rohmer, M.-M.; Welter, R. Angew. Chem., Int. Ed. 2004, 43, 6120.

[4] Goto, E.; Begum, R. A.; Zhan, S.; Tanase, T.; Tanigaki, K.; Sakai, K. Angew. Chem., Int. Ed. 2004, 43, 5029.

[5] Berry, J. F.; Cotton, F. A.; Murillo, C. A. Organometallics 2004, 23, 2503.

[6] Berry, J. F. Metal-Metal Bonding, Ed.: Gerard, P., Springer, Germany, 2010, pp. $1 \sim 28$.

[7] Berry, J. F. Multiple Bonds Between Metal Atoms, Eds.: Cotton, F. A.; Murillo, C. A.; Walton, R. A., Springer, New York, 2005, pp. $669 \sim 706$.

[8] Yeh, C. Y.; Wang, C. C.; Chen, Y. H.; Peng, S. M. Redox Systems under Nano-space Control, Ed.: Hirao, T., Springer, Germany, 2006, pp. $85 \sim 117$.

[9] Liu, I. P.; Chen, C. H.; Chen, C. F.; Lee, G. H.; Peng, S. M. Chem. Commun. 2009, $5,577$.

[10] Huang, X.; Tan, Y.; Xu, X.; Xu, Z. Acta Chim. Sinica 2012, 70, 1979. (黄晓, 谭芗, 许旋, 徐志广, 化学学报, 2012, 70, 1979.)

[11] Tsai, T. W.; Huang, Q. R.; Peng, S. M.; Jin, B. Y. J. Phys. Chem. C 2010, $114,3641$.

[12] Chen, I. W.; Fu, M. D.; Tseng, W. H.; Yu, J. Y.; Wu, S. H.; Ku, C. J.; Chen, C. H.; Peng, S. M. Angew. Chem., Int. Ed. 2006, 45, 5814.

[13] Georgiev, V. P.; McGrady, J. E. Inorg. Chem. 2010, 49, 5591.

[14] Georgiev, V. P.; McGrady, J. E. J. Am. Chem. Soc. 2011, 133, 12590.

[15] Georgiev, V. P.; Mohan, P. J.; DeBrincat, D.; McGrady, J. E. Coord. Chem. Rev. 2013, 257, 290.

[16] Georgiev, V. P.; Sameera, W. M. C.; McGrady, J. E. J. Phys. Chem. C 2012, 116, 20163

[17] Weng, T. T.; DeBrincat, D.; Arcisauskaite, V.; McGrady, J. E. Inorg. Chem. Front. 2014, $1,468$.

[18] te Velde, G.; Bickelhaupt, F. M.; van Gisbergen, S. J. A.; Fonseca Guerra, C.; Baerends, E. J.; Snijders, J. G.; Ziegler, T. J. Comp. Chem. 2001, 22, 931.

[19] Fonseca Guerra, C.; Snijders, J. G.; te Velde G.; Baerends, E. J. Theor. Chem. Acc. 1998, 99, 391.

[20] ADF2012, SCM, Theoretical Chemistry, Vrije Universiteit, Amsterdam, The Netherlands, http: //www. scm. com.

[21] Datta, S. Electronic Transport in Mesoscopic Systems, Cambridge University Press, Cambridge, England, 1995, pp. 293 339 .

[22] OpenMX webpage, http: //www. openmx-square. org/.

[23] Ozaki, T. Phys. Rev. B 2003, 67, 155108.

[24] Berry, J. F.; Cotton, F. A.; Lu, T.; Murillo, C. A.; Roberts, B. K.; Wang, X. J. Am. Chem. Soc. 2004, 126, 7082.

[25] Kondo, H.; Kino, H.; Nara, J.; Ozaki, T.; Ohno, T. Phys. Rev. B 2006, 73, 235323 .

[26] Kondo, H.; Kino, H.; Nara, J.; Ohno, T. Appl. Surf. Sci. 2008, 254, 7985.

[27] Chen, J.; Reed, M. A.; Rawlett, A. M.; Tour, J. M. Science 1999, $286,1550$.

[28] Makinistian, L.; Albanesi, E. A. Appl. Phys. A 2013, 111, 923.

(Zhao, X.) 